If $s_{n}(x)$ is the $n$-th partial sum of the frourier series of $f$, then, observing that $I_{n, u}[f]-s_{n}=I_{n, u}\left[f-s_{n}\right]$, we have

$$
\frac{1}{4 \pi^{2}} \int_{0}^{2 \pi} \int_{0}^{2 \pi}\left|I_{n, u}(x, f)-s_{n}(x)\right|^{2} d u d x=2 \sum_{\lambda=n+1}^{\infty}\left|\gamma_{\lambda}\right|^{2} .
$$

Thence we easily deduce the inequality

$$
\sum_{n=0}^{\infty} \int_{0}^{2 \pi} \int_{0}^{2 \pi}\left|I_{n, u}-s_{n}\right|^{2} d u d x \leqslant C \sum_{\lambda=1}^{\infty} \lambda\left|\gamma_{\lambda}\right|^{2}
$$

where $C$ is an absolute constant. Similarly, if $n_{k+1} / n_{\eta l}>q>1$ and $n_{1} \geqslant 1$,

$$
\sum_{k=1}^{\infty} \int_{0}^{2 \pi} \int_{0}^{2 \pi}\left|I_{n_{k}, u}-s_{n_{k}}\right|^{2} d u d x \leqslant \sigma_{q} \sum_{\lambda=2}^{\infty}\left|\gamma_{\lambda}\right|^{2} \log \lambda,
$$

with $O_{q}$ depending on $q$ only.

The first of these inequalities shows that, if $\Sigma \lambda\left|\gamma_{\lambda}\right|^{2}<\infty$, then, for almost every translation $u$, the sequence $I_{n, u}(x, f)$ converges to $f(x)$ almost everywhere in the interval $0 \leqslant x \leqslant 2 \pi$. The same may be said of the lacunary sequence $I_{n_{k}, u}[f]$, provided that the series $\Sigma\left|\gamma_{\lambda}\right|^{2} \log \lambda$ converges.

\title{
Bibliography.
}

Hardy, G. H., Littlewood, J. E., Polya, G., [1] Inequalitios, Cun. bridge, 1934.

Jackson, D., [1], The Theory of Approximation.

Jessen, B., [1], On the approximation of Lebesgue integrals by Riemann sums, Annals of Mathematics, 35 (1934), 248-251..

Kolmogoroff, A., [1], Sur les fonctions harmoniques conjuguées at les séries de Fourier, Fiund. Math., 7 (1935), 23-28.

Marcinkiewicz, J., [1], Sur l'interpolation, Studia Math. 6 (1936).

[2], Quelques remarques sur l'interpolation, (to appear in Acta Szeged). 218-244.

[2], Sur les maxima des formes billinéaires et sur les tonctionnelles linéaires. Acta Math., 49 (1926), 465-497.

de Ia Vallée Poussin [1], Leçons sur l'approximation, Paris 1919, Zygmund, A., [1], A remark on conjugate series, Proo. London Math, Soc. 34 (1932), 392-400.

[2], Trigonometrical Series (Monografje Matematyczne V), Warszawa-Lwów, 1935.

[3], Sur les fonctions conjuguées, Fund. Math. 13 (1929), 284-303.

\section{Sur la géométrisation des types d'ordre dénombrable ${ }^{1}$ ).}

Par

\author{
Cas im ir Kuratowski (Warszawa).
}

D'après les théorèmes classiques de la Théorie des Ensembles, on peut faire correspondre d'une façon bien déterminée aux types d'ordre d'ensembles dénombrables ordonnés certains ensembles de nombres réels: c'est que chaque ensemble ordonné dénombrable est semblable à un sous-ensemble de l'ensemble des nombres rationnels rangés selon leur grandeur (et même, à une infinité de tels ensembles); et qu'en outre, chaque ensemble composé de nombres rationnels peut être remplacé par un nombre réel, notamment, par le nombre réel qui lui vient correspondre dans la correspondance biunivoque entre la famille de tous les ensembles composés de nombres rationnels et l'ensemble de tous les nombres réels.

Nous allons réaliser cette interprétation géométrique des types d'ordre dénombrable à l'aide de la méthode suivante, due à $M$. Lebesgue et qui paraît être la plus simple possible ${ }^{2}$ ).

Imaginons d'abord l'ensemble des nombres rationnels de l'intervalle 01 rangé en une suite infinie bien déterminée (composée d'éléments différents)

$$
r_{1}, r_{2}, \ldots r_{n}, \ldots
$$

Soit $t$ un élément de l'ensemble $\mathcal{e}$ non-dense de Cantor:

$$
t=\frac{t^{1}}{3}+\frac{t^{2}}{9}+\frac{t^{3}}{27}+\ldots \quad\left(t^{n}=0 \text { ou } 2\right) .
$$

1) Présenté à la Soc. Pol. de Math., Section de Varsovie, le 9. X. 1936. 2) Journal de Math. 1905 (chap. VIII). 
Désignons par $M_{t}$ l'ensemble des nombres rationnels $r_{n}$ tels que $t^{n}=2$. Evidemment, la fonction $M_{t}$ établit une correspondance biunivoque entre les éléments de l'ensemble $\mathcal{e}$ et les sous-ensembles de la suite (1).

Soit $\bar{t}$ le type d'ordre de l'ensemble $M_{t}$ (ordonné selon la grandeur eroissante de ses éléments). L'interprétation géométrique des types ordinaux consiste à remplacer le type ordinal $\tau$ par l'ensemble des nombres $t$ tels que $\bar{t}=\tau$; en symboles: par l'ensemble $\underset{t}{E}(\bar{t}=\tau)$.

Ainsi à un ensemble $\Phi$ de types ordinaux correspond l'ensemble de nombres réels $\underset{t}{E}(\vec{t} \in \Phi)$. Autrement dit, à une propriété des types ordinaux (ou encore: à une fonction propositionnelle $\varphi(v)$ de variable $\tau$ ) correspond une propriété des nombres réels: à savoir, celle d'être un nombre $t$ tel que $t$ jouit de la propriétés considérée (c. à d. à $\varphi(\tau)$ correspond la fonction propositionnelle $\varphi(t)$ de van riable réelle). $\mathrm{Si}$, par exemple, $\Phi$ est l'ensemble des nombres ordinaux (= types de bon ordre), l'ensemble des nombres réels qui lui vient correspondre est celui des $t$ tels que $t<\Omega$.

D'une façon analogue, à une relation entre les types ordinaux correspond une relation entre les éléments de l'ensemble de Cantor: à la relation $\varphi(t, \sigma)$ correspond la relation $\varphi(\bar{t}, \bar{s})$. Autrement dit, si $\widetilde{c}$ dénote l'ensemble de tous les types d'ordre dénombrable et $\widetilde{\tau} \times \widetilde{\tau}$, ou $\widetilde{\tau}^{2}$, l'ensemble de tous les couples des éléments de $\widetilde{\varepsilon}$ (types ordinaux "complexes"), - à chaque ensemble $\Phi \subset \widetilde{C}^{2}$ correspond l'ensemble $\underset{t s}{E}[(\bar{t}, \bar{s}) \in \Phi]$, contenu dans $\mathfrak{C}^{2}$. Ainsi, par exemple, à la relation $x=\sigma$ correspond la relation $\bar{t}=\bar{s}$; ce qui revient à dire qu'à l'ensemble $\Phi=\underset{\tau \sigma}{E}(\tau=\sigma)$ correspond l'ensemble „plan" $\underset{t s}{E}(\bar{t}=\bar{s})$.

D'une façon tout-à-fait générale, à chaque ensemble $\Phi \subset \widetilde{C}^{n}$

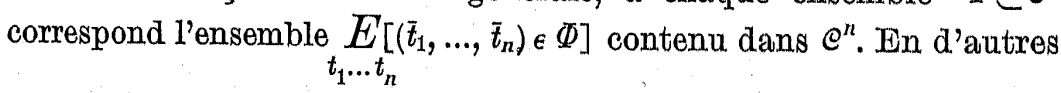
termes, à la fonction propositionnelle $\varphi\left(\tau_{1}, \ldots, v_{n}\right)$ correspond $\varphi\left(\bar{t}_{1}, \ldots, \bar{t}_{n}\right)$.

La définition suivante s'impose:
Definition. L'ensemble $\Phi \subset \widetilde{C}^{n}$ est dit de classe projective $P_{n}$

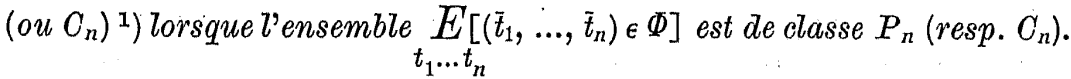

En d'autres termes, la fonotion propositionnelle $\varphi\left(\boldsymbol{\tau}_{1}, \ldots, \boldsymbol{\tau}_{n}\right)$ de $n$ types ordinaux est dite de classe $P_{n}$ (ou $C_{n}$ ) lorsque la fonction propositionnelle $\varphi\left(\bar{t}_{1}, \ldots, \bar{t}_{n}\right)$ de $n$ variaibles réelles est de cette classe.

Le but de cet ouvrage est l'étude des notions (des fonctions propositionnelles) de la théorie des types d'ordre dénombrable au point de vue de leur classe projective. Comme on verra, toutes les notions habituellement considérées dans la théorie des types ordinaux sont projectives; de plus, dans la théorie des nombres ordinaux, elles sont; en général, de classe $O A{ }^{2}$ ).

Plusieurs cas particuliers du problème ainsi posé ont été considérés dans des différentes recherches. Ainsi par exemple, le théorème important d'après lequel l'ensemble des $t$ tels que $\bar{t}<\Omega$ est non borelien ${ }^{3}$ ) s'énonce en termes employés ici de cette façon: l'ensemble des nombres ordinaux n'est pas borelien. Un autre théorème qui se rattache au précédent est que, pour chaque $\alpha<\Omega$, l'ensemble $E(\bar{t}=\alpha)$ est borelien $\left.{ }^{4}\right)$; cela veut dire que chaque nombre ordinal individuel, considéré comme ensemble formé d'un seul élément, est borelien.

1) Les ensembles boreliens (= ensembles qui s'obtiennent des ensembles fermés à l'aide des opérations de l'addition et de la multiplication dénombrables indéfiniment répétées) constituent la classe $P_{0}$ ainsi que $O_{0}$. Les ensembles de classe $P_{n}$ sont les images continues des ensembles de classe $O_{n-1}$. Ceux de classe $O_{n}$ sont les complémentaires des ensembles de classe $P_{n}$. En particulier, les ensembles $P_{1}$ sont nommés ensembles analytiques (ou ensembles $A$ ), ceux de classe $O_{1}$ sont nommés ensembles $O A$.

Comme on sait, les ensembles boreliens se partagent en classes $F_{\alpha}$ et $G_{\alpha}$; on pourrait nommer l'ensemble $\Phi$ ensemble de classe $F_{\alpha}$ (ou $G_{\alpha}$ ) lorsque $E\left[\left(\bar{t}_{1}, \ldots, \bar{t}_{n}\right) \in \Phi\right]$ est de telle classe. $t_{1} \ldots t_{n}$

2) L'existence des ensembles composés de nombres ordinaux qui ne sont pas des ensembles de classe $O A$, qui ne sont même pas projectifs, résulte (en admettant l'hypothèse $d u$ continu) du fait que la famille de tous les sous-ensembles de l'ensemble des nombres ordinaux est de puissance $2^{\mathrm{N}_{1}}$ tandis que celle des ensembles projectifs est de puissance du continu.

Le problème reste ouvert de définir effectivement un ensemble de nombres ordinaux qui ne soit pas de classe $O A$.

3) Voir N. Lusin et W. Sierpiński, Journ. de Math. 1923, p. 53.

4) Voir N. Lusin et W. Sierpiński, C. R. Paris t. 175 (1922), p. 357. 
Le fait que l'inégalité $x \leqslant \sigma$ est analytiques constitue, on résulité, la base de la démonstration da "deuxième principe "do M.. I Lusin "). Ajoutons enfin que la projectivité des ensembles des types pairs et limites intervient dans les recherches récentes sur la projectivité des ensembles définis à l'aide de l'induction transfinies ${ }^{2}$ ).

Dans une note qui va suivre, jo vais appliquer la thésorie développée ici aux suites transfinies projectives d'ensembles (dont j'ai signalé récemment la définition) ${ }^{3}$ ) ainsi qu'à la théorie des onsembles projectifs en général.

\section{\$1. Types d'ordre dénombrable.}

1. Définitions des notions fondamentales. Dans co qui va suivre $M, N$ et $P$ désignent des sous-ensembles variables de l'ensemble des nombres rationnels de l'intervalle 01. Lo typo d'orilro de $M$ est désigné par $\bar{M}$. Les symboles $z=\left\{z^{1}, z^{2}, \ldots\right\}$ ot $\mathfrak{y}=\left\{\mathfrak{y}^{1}, y^{2}, \ldots\right\}$ dénotent des suites variables de nombres réels (e. à d. z st y sont des points de l'espace de Fréchet). Nous nous servirons on outre des notations logiques, habituellement employées ${ }^{4}$ ).

Définition 1. Type d'ordre limite (c. à d. typo d'ordre sans dernier élément). $L$ désignant l'ensemble des types limites, on a

$$
\left\{\bar{M}_{\in} L\right\} \equiv \prod_{n} \sum_{h}\left(r_{n} \in M\right) \rightarrow\left(r_{n}<r_{k} \in M\right)
$$

Déf. 2. Identite. $\{\bar{M}=\bar{N}\} \equiv \sum_{\mathfrak{z})}\left\{\prod_{n}\left[\left(\mathfrak{z}^{n} \in \mathbb{M}\right)\left(\mathfrak{b}^{n} \in N\right)\right]\right.$. $\left.\left.\cdot \prod_{k}\left[\left(r_{k} \in M\right) \rightarrow \sum_{n}\left(r_{k}=z^{n}\right)\right]\left[\left(r_{k} \in N\right) \rightarrow \sum_{n}^{n}\left(r_{k}=y^{n}\right)\right] \cdot \prod_{\| /}\left[z^{l}<z^{\prime}\right)=\left(\mathfrak{y}^{\prime}<\mathfrak{y}^{\prime}\right)\right]\right]$.

Cette définition signifie, en effet, que l'on peut xanger les éléments de $M$ ainsi que ceux de $N$ en deux suites $z=\left\{z^{1}, z^{2}, \ldots\right\}$ et $\mathfrak{y}=\left\{\mathfrak{y}^{1}, \mathfrak{y}^{2}, \ldots\right\}$ de façon que l'inégalité $z^{t}<z^{\prime}$ équivale à $y^{i}<\mathfrak{y}^{\prime}$; cela veut dire que les ensembles $M$ et $N$ sont semblables.

1) Voir N. Lusin, Ensembles analytiques, Paris 1930, p. 210 ot ma Topologie I, Varsovie 1933, p. 258.

2) Cf. ma note dans Fund. Math. 27, p. 275 ot la note de M. J. v. Noumann et moi dans Annals of Math. (a paraitre).

$\left.{ }^{3}\right)$ dans ma conférence au Congrès topologique de Moscou (1935).

4) en particulier des opérateurs $\sum_{x}$, qui signifie "il existe un $x$ tel que..." et $\prod_{x}$, qui signifie "quel que soit $x$, on a..." Voir Topologie $I, \S 1$.
Def. 3. Addition. $\{\bar{M}=\bar{N}+\bar{P}\} \equiv \sum_{\mathfrak{z} \mathfrak{n}} \prod_{n}\left[\left(\mathfrak{\jmath}^{n} \in M\right)\left(\left(\mathfrak{y}^{n} \in N\right)+\left(\mathfrak{y}^{n}-2 \in P\right)\right)\right]$.

$$
\begin{gathered}
\cdot \prod_{k}\left[\left(r_{k} \in M\right) \rightarrow \sum_{n}\left(r_{k}=z^{n}\right)\right]\left[\left(r_{k} \in N\right) \rightarrow \sum_{n}\left(r_{k}=\mathfrak{y}^{n}\right)\right]\left[\left(r_{k} \in P\right) \rightarrow \sum_{n}\left(r_{k}=\mathfrak{y}^{n}-2\right)\right] . \\
\left.\cdot \prod_{i j}\left[\left(\mathfrak{z}^{i}<\right\}^{j}\right) \equiv\left(\mathfrak{y}^{i}<\mathfrak{y}^{j}\right)\right] .
\end{gathered}
$$

Dêf. 4. Multiplication.

$$
\begin{gathered}
\{\bar{M}=\bar{N} \cdot \bar{P}\} \equiv \sum_{\mathfrak{y} \mathfrak{w})} \prod_{n}\left[\left(\mathfrak{z}^{n} \in M\right)\left(\mathfrak{y}^{n} \in N\right)\left(\mathfrak{w}^{n} \in P\right)\right] . \\
\cdot \prod_{k}\left[\left(r_{k} \in M\right) \rightarrow \sum_{n}\left(r_{k}=\mathfrak{z}^{n}\right)\right]\left[\left(\mathfrak{r}_{k} \in N\right) \rightarrow \sum_{n}\left(\mathfrak{r}_{k}=\mathfrak{y}^{n}\right)\right]\left[\left(r_{k} \in P\right) \rightarrow \sum_{n}\left(r_{k}=\mathfrak{w}^{n}\right)\right] . \\
\cdot \prod_{i j}\left[\left(\mathfrak{z}^{i}<\mathfrak{z}^{j}\right) \equiv\left(\mathfrak{w}^{i}<\mathfrak{w}^{j}\right)+\left(\mathfrak{w}^{i}=\mathfrak{w}^{j}\right)\left(\mathfrak{y}^{i}<\mathfrak{y}^{j}\right)\right] .
\end{gathered}
$$

\section{Dêf. 5. Inégalités.}

$\{\bar{N}\langle\bar{M}\} \equiv\{N$ est semblable à un sous-ensemble de $M\} \equiv$ $\left.\equiv \sum_{\mathfrak{z} \mathfrak{n}} \prod_{k}\left[\left(r_{k} \in N\right) \rightarrow \sum_{n}\left(r_{k}=\mathfrak{y}^{n}\right)\right]\left(\mathfrak{z}^{k} \in M\right) \prod_{i j}\left[\left(\mathfrak{y}^{i}<\mathfrak{y}^{j}\right) \rightarrow\left(\mathfrak{z}^{i}<\mathfrak{z}^{j}\right)\right]{ }^{1}\right)$.

En outre

$$
(\tau \leqslant \sigma) \equiv \sum_{\varrho}(\sigma=\tau+\varrho), \quad(\tau<\sigma) \equiv(\tau+1 \leqslant \sigma) .
$$

En vue des applications ajoutons les définitions suivantes:

$(\bar{M}$ est dense $) \equiv \prod_{n m} \sum_{k}\left(r_{n}, r_{m} \in M\right)\left(r_{n}<r_{m}\right) \rightarrow\left(r_{n}<r_{k}<r_{m}\right)\left(r_{k} \in M\right)$

$(\bar{M}$ est somme d'un type limite et de $n) \equiv$

$$
\begin{gathered}
=\sum_{k_{1} \ldots k_{n}}\left\{( r _ { k _ { 1 } } \ldots r _ { k _ { n } } \in M ) ( r _ { k _ { 1 } } < \ldots < r _ { k _ { n } } ) \cdot \prod _ { m } \left[\left(\left(r_{m} \in M\right) \prod_{i \leqslant n}\left(m \neq k_{i}\right)\right) \rightarrow\right.\right. \\
\left.\rightarrow \sum_{j}\left(r_{m}<r_{j} \in M\right)\left(r_{j}<r_{k_{1}}\right]\right) .
\end{gathered}
$$

$(\bar{M}$ est pair $) \equiv$ (il existe un $n$ tel que $\bar{M}=$ type limite $+2 n)^{2}$ ). Convenons aussi que

$$
(\tau=\sigma-1) \equiv[(\sigma \in L)(\tau=\sigma)+(\sigma=\tau+1)] .
$$

2. Evaluation de la classe borelienne ou projective dans la thérorie des types d'ordre dénombrable.

Théorème 1. L'ensemble des types d'ordre limite (ainsi que celui des types denses et celui des types pairs) est borelien. Les relations $\tau=\sigma, \tau \leqslant \sigma, \tau<\sigma, \tau \prec \sigma, \tau=\sigma+\varrho, \tau=\sigma . \varrho$ et $\tau=\sigma-1$ sont analytiques.

Démonstration. Remplaçons, dans la déf. $1, r_{n} \in M$ par $t_{n}=2$. Il vient:

$$
\{\bar{t} \epsilon L\} \equiv\left\{\bar{M}_{t} \in L\right\} \equiv \prod_{n} \sum_{k}\left(t^{n}=2\right) \rightarrow\left(r_{n}<r_{k}\right)\left(t^{k}=2\right) .
$$

1) Cf. le renvoi $\left.{ }^{1}\right)$ p. 170.

2) Cettie dernière définition n'est considérée d'habitude que dans le cas où $M$ est bien ordonné. 
L'ensemble $\underset{t}{E}\left(t^{n}=2\right)$ étant simultanémenti formé ot ouvert (dans l'ensemble de Cantor), l'ensemble $L$ est borelien (de classe $G_{\delta}$ ).

Un raisonnement analogue conduit à la conclusion que les types d'ordre dense et pair sont boreliens.

Pour démontrer que la relation $r=\sigma$ est analytique, ce qui veut dire que l'ensemble $\underset{t s}{E}(\bar{t}=\bar{s})$ est analytique, on se sert de l'équivalence

$$
\left(\gamma^{n} \in M_{t}\right) \equiv \sum_{k}\left(r_{k}=3^{n}\right)\left(r_{k} \in M_{t}\right) \equiv \sum_{k}^{\top}\left(r_{k}=\gamma^{n}\right)\left(t^{k}=2\right) .
$$

Les ensembles $\underset{z}{E}\left(r_{k}=z^{n}\right)$ et $E_{z}^{\top}\left(z^{k}<\gamma^{n}\right)$ étant fermés pour $k$ et $n$ fixes, on en conclut ${ }^{1}$ ) que l'ensemble

$$
Q=\underset{t_{s 3 \mathfrak{z}}}{E}\left\{\prod_{n} \sum_{k l}\left(r_{k}=8^{n}\right)\left(r_{l}=\mathfrak{y}^{n}\right)\left(t^{k}=2=s^{l}\right)\right.
$$

$\left.\cdot \prod_{k}\left[\left(t^{k}=2\right) \rightarrow \sum_{n}\left(r_{k}=z^{n}\right)\right]\left[\left(s^{k}=2\right) \rightarrow \sum_{n}\left(r_{k}=y^{n}\right)\right] \cdot \prod_{l l}\left[\left(z^{l}<z^{\prime}\right) \equiv\left(y^{l}<y^{\prime}\right)\right)\right\}$ est borelien. L'ensemble $\underset{t s}{E}(\bar{t}=\bar{s})=\underset{t s}{E}\left(\bar{M}_{t}=\bar{M}_{s}\right)=\sum_{z y} \underset{t s}{E}[(t s z y) \epsilon Q]$, comme projection de $Q$, est donc analytique.

D'une façon tout-à-fait analogue, on démontre que les relations $\tau\langle\sigma, \quad \tau=\sigma+\varrho$ et $\tau=\sigma \cdot \varrho$ sont analytiques. De là on conclut en vertu. des équivalences

$$
(\bar{t} \leqslant \bar{s}) \equiv \sum_{v}(\bar{s}=\bar{t}+\bar{v}) \quad \text { et } \quad(\bar{t}<\bar{s})=\sum_{n}(\bar{v}=1)(\bar{t}+\bar{v} \leqslant \bar{s})
$$

que les relations $\tau \leqslant \sigma$ et $\tau<\sigma$ sont analytiques (c'est d'ailleurs une conséquence de la règle 4) qui va suivre). Il en est de même de la relation $\tau=\sigma-1$.

L'analycité des relations fondamentales qui vient d'être établie implique la projectivité des notions habituellement considérées dans la théorie des types d'ordre dénombrable. Car chaque fonction propositionnelle $\varphi(\tau, \sigma, \ldots)$ qui s'obtient à partir des fonctions propositionnelles projectives en appliquant les opérations de l'algèbre de la logique: addition, multiplication, négation, opérateurs $\sum_{t}$ et $\prod_{t},-$

1) Pour la méthode d'évaluation de la classe borelienne ou projective d'une fonction propositionnelle, voir Topologie $I, \mathrm{pp} .168$ ot 243 ou derx notes de M. Tarski et moi dans Fund. Math. 17 (1931). est projective. L'évaluation de sa classe projective s'effectue à l'aide de la même méthode que l'on emploie dans le cas où, au lieu des variables $\tau, \sigma$, etc., on a à considérer des variables réelles (ou plus généralement des points d'un espace complet séparable).

On a, en particulier, les règles suivantes d'évaluation de la classe projective d'une fonction propositionnelle $\varphi(\tau)$ :

1) Si $\varphi(\tau)$ est de classe $P_{n}$, sa négation $\varphi^{\prime}(\boldsymbol{\tau})$ est de classe $C_{n}$. Car $\underset{t}{E} \varphi^{\prime}(\bar{t})=\widetilde{c}-\underset{t}{E} \varphi(\bar{t})$.

2) Si $\varphi(\tau)$ est de classe $P_{n}\left(\right.$ ou $\left.C_{n}\right)$ dans l'espace $\widetilde{\leftarrow}$, il en est de

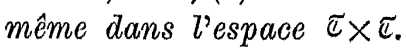

$$
\text { Car } \underset{t s}{E} \varphi(\bar{l})=[\underset{t}{E} \varphi(\bar{t})] \times \widetilde{\varangle} \text {. }
$$

3) Si les fonctions $\varphi_{1}(\tau), \varphi_{2}(\tau), \ldots$ sont de classe $P_{n}$ (ou $C_{n}$ ), $i l$ en est de même des fonctions $\sum_{k=1}^{\infty} \varphi_{k}(\tau)$ et $\prod_{k=1}^{\infty} \varphi_{k}(\tau)$.

$$
\operatorname{Car} \underset{t}{E} \sum_{k} \varphi_{k}(\bar{t})=\sum_{k} \underset{t}{E} \varphi_{k}(\bar{t}) \text {. }
$$

4) $\varphi(\tau, \sigma)$ étant de classe $P_{n}$, la fonction $\psi(r)=\sum_{\sigma} \varphi(\tau, \sigma)$ l'est également; la fonction $\chi(\tau)=\prod_{\sigma} \varphi(\tau, \sigma)$ est de classe $C_{n+1}$.

En effet, à chaque $\sigma$ correspond un $s$ tel que $\bar{s}=\sigma$. Donc, $\sum_{\sigma} \varphi(\tau, \sigma) \equiv \sum_{\boldsymbol{s}} q(\tau, \bar{s})$, d'où, $\psi(\bar{t}) \equiv \sum_{\boldsymbol{s}} \varphi(\bar{t}, \bar{s})$, ce qui implique que l'ensemble $\underset{t}{E} \psi(\bar{t})$ est de classe $P_{n}$, comme projection de l'ensemble $\underset{t s}{E} \varphi(\bar{t}, \bar{s})$.

5) $\varphi(\tau, \sigma)$ étant de classe $P_{n}\left(\right.$ ou $\left.C_{n}\right)$, il en est de même de la fonction $\psi(\tau)=\varphi\left(\tau, \sigma_{0}\right)$, pour $\sigma_{0}$ fixe $\left.{ }^{\mathbb{1}}\right)$.

1) On en conclut on vertu du théor. I que l'ensemble $\underset{t}{E}(\bar{t}=\sigma)$ est analytique quel que soit $\sigma$. Le problème s'impose de reconnaître si cet ensemble n'est pas nécessairement borelien? Il en est ainsi, si l'on fait l'hypothèse supplémentaire que $\sigma$ est un nombre ordinal. De plus, on peut démontrer que la classe borelienne de l'ensemble $\underset{t}{E}(\bar{t}=\alpha)$ est illimitée lorsque $\alpha$ parcourt l'ensemble des nombres ordinaux. Il en résulte aussitôt que l'ensemble $\underset{t s}{E}(\bar{t}=\bar{s})$ n'est pas borelien. Autrement dit, l'égalité $x=\sigma$ est une relation analytique non borelienne. 
Soit, en effet, $s_{0}$ un nombre tiel que $\bar{s}_{0}=\sigma_{0}$. L'ensemble $L_{t}^{\gamma} \psi(\bar{t})$ coincide avec l'intersection de l'ensemble $\underset{t s}{\mathbb{E}} \varphi(\bar{t}, \bar{s})$ par la droites $s=s_{0}$. Car on a

$$
\psi(\bar{t}) \cdot\left(s=s_{0}\right) \equiv \varphi\left(\bar{t}, \sigma_{0}\right) \cdot\left(s=s_{0}\right) \equiv \varphi(\bar{t}, \bar{s}) \cdot\left(s=s_{0}\right) .
$$

6) $\varphi(\tau, \sigma)$ étant de classe $P_{n}$ (ou $\left.O_{n}\right)$, il en est de même de la fonction $\varphi(\tau, \tau)$.

En effet, en projetant la diagonale $\underset{t s}{E}(t=s)$ sur l'axe des abscisses, l'ensemble $\underset{t}{E} \varphi(\bar{t}, \bar{t})$ vient correspondre à l'onsemble $\underset{t s}{E}[\varphi(\bar{t}, \bar{s}) \cdot(t=s)]$, qui est évidemment de la môme classe que l'ensemble $\underset{t s}{E} \varphi(\bar{t}, \bar{s})$.

Outre les règles précédentes, qui correspondent aux rògles générales de l'évaluation de la classe projective, on a le thécorème suivant.

Théorème 2. Les opérations $\prod_{\sigma<\tau}$ et $\sum_{\sigma<t} n^{\prime}$ altèrent pas la olasse projective de la fonction propositionnelle da laquelle elles sont appliquées.

Autrement dit, si la fonction $\varphi(\sigma)$ est de classe $P_{n}$ (ou $C_{n}$ ), il en est de même des fonctions

$$
\psi(\tau) \equiv \sum_{\sigma<\tau} \varphi(\sigma) \quad \text { et } \quad \chi(\tau) \equiv \prod_{\sigma<\tau} \varphi(\sigma)
$$

Démonstration. Définissons d'abord une fonction auxiliaire $t^{(n)}$ (pour $t \epsilon C$ ), comme suit: si $t^{n}=0, t^{(n)}=0$; si $t^{n}=2, M_{t^{(n)}}=M_{t} \cdot \underbrace{}_{s}\left(s<r_{n}\right)$. En symboles:

$$
\left\{u=t^{(n)}\right\} \equiv \prod_{k}\left\{\left(u^{k}=2\right) \equiv\left(t^{k}=2=t^{n}\right)\left(r_{k}<r_{n}\right)\right\} .
$$

Evidemment la fonction $t^{(n)}$, pour $n$ fixe, est continue. En outre, pour $t$ fixe $\neq 0$, la suite $\overline{t^{(1)}}, \overline{t^{(2)}}, \ldots$ parcourt tous les types ordinaux $<\bar{t}$. On a en effet

$$
M_{t}=M_{t} \cdot \underset{s}{E}\left(s \leqslant r_{n}\right)+M_{t} \cdot \underset{s}{E}\left(s>r_{n}\right)
$$

et pour $r_{n} \in M_{t}$, l'ensemble $M_{t} \cdot E_{s}\left(s \leqslant r_{n}\right)$ a le type $\overline{t^{(n)}}+1$.
De là on conclut, d'une part, que $t^{(n)}<\bar{t}$, quel que soit $n$, et d'autre part, qu'à chaque $\sigma<\tau$ correspond un $n$ tel que $\overline{t^{(n)}}=\sigma$; à savoir, l'indice $n$ tel que $r_{n} \in M_{t}$ et que $\overline{M_{t} \cdot E\left(s \leqslant r_{n}\right)}=\sigma+1$.

Cela étant, il vient $\psi(\bar{t}) \equiv(\bar{t} \neq 0) \sum_{n=1}^{\infty} \psi\left(\overline{t^{(n)}}\right)$.

La fonction $t^{(n)}$ étant continue, l'ensemble $\underset{t}{E} \varphi\left(t^{(\bar{n})}\right)$ est de la même classe que $\underset{t}{E} \varphi(\bar{t})$. L'addition $\sum_{n}$ étant dénombrable, il en est encore de même de l'ensemble $\underset{t}{E} \psi(\bar{t})$.

La fonction $\psi$ est donc de la même classe que $\varphi$. Il en résulte aussitôt que cette classe contient aussi la fonction $\chi$.

3. Types ordinaux, ,complexes“. Un couple $(r, \sigma)$ de types ordinaux, c. à d. un élément de $\widetilde{\mathbb{C}}^{2}$, peut être nommé type ordinal complexe. D'une façon plus générale, nous appellerons type ordinal complexe toute suite finie ou infinie (simple) $\Delta=\left[\Delta^{1}, \Delta^{2}, \ldots\right]$ de types ordinaux, c. à d. tout élément de $\widetilde{\mathbb{C}}^{\aleph_{0}}$.

Evidemment chaque fonction propositionnelle de plusieurs variables $\varphi\left(\tau_{1}, \ldots, \tau_{n}\right)$ peut être considérée comme fonction propositionnelle d'une seule variable complexe $\Delta=\left[\Delta^{1}, \ldots, \Delta^{n}\right]$. Etant donnée une suite finie ou infinie de points de l'ensemble de Cantor $\delta=\left[\widehat{b}^{1}, \delta^{2}, \ldots\right]$, autrement dit, étant donné un point o de l'ensemble $\mathfrak{C} \times \mathfrak{e} \times \ldots \times \mathfrak{C}$ (répété $n$ fois) ou bien de l'ensemble $e^{N_{0}}$, convenons de désigner par $\bar{D}$ la suite $\bar{D}^{1}, \bar{D}^{2}, \ldots$ (ainsi, par exemple, au nombre complexe $z=x+i y$ correspond le type ordinal complexe $\bar{z}=(\bar{x}, \bar{y})$ ). Conformément à la définition proposée dans l'Introduction, un ensemble $\Phi$ de types ordinaux complexes sera dit de classe $P_{n}$ (ou $O_{n}$ ) lorsque l'ensemble $E(\bar{\delta} \in \Phi)$ est de telle classe.

Appelons $\Delta^{n}$, ,n-ème coordonnée de $\Delta^{" .}$. La retation $\tau=\Delta^{n}$ est analytique. En d'autres termes, l'ensemble $\underset{t o}{E}\left(\bar{t}=\bar{D}^{n}\right)$ est analytique. Or, ceci est une simple conséquence de l'analycité de l'ensemble $E(\bar{t}=\bar{s})$ et de la continuité de la fonction $s=\delta^{n}$ (la $n$-ième coordonnée. d'un point de l'espace à un nombre fini ou infini de dimensions est. une fonction continue de ce point) ${ }^{1}$ ).

1) Car l'ensemble $E \varphi(x)$ étant analytique et la fonction $x=f(t)$ continue, l'ensemble $\underset{t}{E} \varphi(f(t))$ est analytique. 
Pour les mêmes raisons l'égalité $\Delta=T^{\prime}$ est analytique. Car

$$
\underset{\mathfrak{d g}}{E}(\overline{\mathrm{D}}=\overline{\mathfrak{g}})=\prod_{n=1}^{\infty} E_{\mathfrak{d g}}\left(\overline{\mathrm{D}}^{n}=\overline{\mathrm{g}}^{n}\right) \text {. }
$$

Remarquons, en outre, que l'évaluation de la classe projective dans l'espace $\widetilde{C}^{\text {\$o }}$ s'effectue comme dans le cas considéré auparavant; en particulier, les énoncés 1)-6) du $\mathrm{N}^{0}$ précédent restent valables pour les variables complexes.

4. Fonctions. $\mu(\tau)$ étant une fonction qui fait correspondre à chaque type ordinal $\tau$ (complexe ou non) un type ordinal $\mu(\tau)$ (complexe ou non), nous dirons que octte fonotion est de olasse $P_{n}$ (ou $O_{n}$ ) lorsqu'à cette classe appartient la relation $\sigma=\mu(\tau)$.

Pour que la fonction $\mu(\tau)$ d valeurs complexes soit de olasse $\dot{P}_{n}$ $(n>0)$, il faut et il suffit que chacune des fonctions $\mu^{\mathrm{I}}(r), \mu^{2}(\tau), \ldots$ le soit.

Car, on a

$$
\begin{aligned}
& {\left[\sigma=\mu^{k}(\tau)\right] \equiv \sum_{\Delta}\left(\Delta^{k}=\sigma\right)(\Delta=\mu(\tau))} \\
& [\lrcorner=\mu(\tau)] \equiv \prod_{k} \sum_{\sigma}\left(\Delta^{k}=\sigma\right)\left(\sigma=\mu^{h}(\tau)\right)
\end{aligned}
$$

et la fonction $\Delta^{k}$ est analytique selon $N^{0} 3$.

Théorème. $\mu(\tau)$ étant une fonotion de classe $P_{n}(n>0)$ et $\Phi$ un ensemble de classe $P_{n}$ (ou $O_{n}$ ) de types ordinaun (complexes on non), l'ensemble $\mu^{-1}(\Phi)=\underset{\tau}{E}[\mu(\tau) \in \Phi]$ est de classe $P_{n}\left(\right.$ resp. $\left.O_{n}\right)$.

En particulier, étant données une fonction propositionnelle de plusieurs variables $\varphi\left(\sigma_{1}, \ldots, \sigma_{m}\right)$ de classe $P_{n}$ (ou $\left.O_{n}\right), n>0$, et $m$ fonctions $\mu_{1}(\tau), \ldots, \mu_{m}(\tau)$ de classe $P_{n}$, la fonction propositionnelle $\varphi\left[\mu_{1}\left(\tau_{1}\right), \ldots, \mu_{m}\left(\tau_{m}\right)\right]$ est de classe $P_{n}\left(\right.$ resp. $\left.C_{n}\right)$.

Démonstration. On a, en effet

$$
[\mu(\tau) \in \Phi] \equiv \sum_{\sigma}[\sigma=\mu(\tau)](\sigma \in \Phi)
$$

En supposant $\Phi$ de classe $P_{n}$, on conclut de 4) (p. 173) que l'en.semble $\underset{\tau}{E}\left[\mu(\tau)_{\epsilon} \Phi\right]$ est aussi de classe $P_{n}$. Si $\Phi$ est de classe $C_{n}$, le complémentaire $\Phi^{\prime}$ de $\Phi$ est de classe $P_{n}$, donc $\underset{v}{E}\left[\mu(v) \in \Phi^{\prime}\right]$ est de classe $P_{n}$ et par conséquent son complémentaire, c. à d. l'ensemble $E[\mu(\tau) \in \Phi]$, est de classe $C_{n}$.

En outre, en considérant la suite $\left[\mu_{1}\left(\tau_{1}\right), \ldots, \mu_{m}\left(\tau_{m}\right)\right]$ comme une fonction complexe de variable complexe: $I=v(\Delta)$ où $\nu^{i}(\Delta)=\mu_{i}\left(\Delta^{i}\right)$, $1 \leqslant i \leqslant m$, la fonction $\nu(\Delta)$ est de classe $P_{n}$. En effet, $\nu^{i}(\Delta)$ est de classe $P_{n}$, car

Il vient

$$
\left[\sigma=\nu^{i}(\Delta)\right] \equiv \sum_{\tau}\left(\tau=\Delta^{i}\right)\left(\sigma=\mu_{t}(\tau)\right)
$$

$$
\underset{\tau_{1} \cdots \tau_{m}}{E} \varphi\left[\mu_{1}\left(\tau_{1}\right), \ldots, \mu_{m}\left(\tau_{m}\right)\right]=\underset{\Delta}{E} \varphi[\nu(\Delta)]=\underset{\Delta}{E}\{\nu(\Delta) \in \underset{\Gamma}{E} \varphi(\Gamma)\},
$$

d'où la conclusion demandée, l'ensemble $\underset{\Gamma}{\operatorname{E}} \varphi(T) \underset{\sigma_{1} \ldots \sigma_{m}}{\operatorname{E} \varphi\left(\sigma_{1}, \ldots, \sigma_{m}\right)}$ étant de classe $P_{n}$ (ou $C_{n}$ ) par hypothèse.

Corollaives. Si la fonction $\mu(\tau)$ est de classe $P_{n}(n>0)$, il en est de même des inégalités $\sigma \leqslant \mu(r)$ et $\sigma<\mu(\tau)$.

La superposition de deux fonctions de classe $P_{n}$ est une fonction de classe $P_{n}$.

5. Relativisation. Soit $\Psi$ un ensemble de types d'ordre donné (ou, plus généralement, $\Psi \subset T^{n}$ ou $\Psi \subset T^{\mathbb{N}_{0}}$ ). Nous dirons qu'un sous-ensemble $\Phi$ de $\Psi$ est relativement (par rapport à $\Psi$ ) de classe $P_{n}$ (ou $C_{n}$ ) lorsque $\Phi$ est la partie commune de $\Psi$ et d'un ensemble de classe $P_{n}$ (resp. $C_{n}$ ).

Ainsi une fonction propositionnelle $\varphi\left(\tau_{1}, \ldots, \tau_{m}\right)$ dont les variables parcourent $\Psi$ est relativement de classe $P_{n}$ (ou $C_{n}$ ) lorsqu'elle se laisse "prolonger" en une fonction propositionnelle $\varphi^{*}\left(\tau_{1}, \ldots, \tau_{n}\right)$ de telle classe; plus précisément, lorsqu'on a l'équivalence

$$
\varphi^{*}\left(\tau_{1}, \ldots, \tau_{m}\right)\left(\tau_{1}, \ldots, \tau_{m} \in \Psi\right) \equiv \varphi\left(\tau_{1}, \ldots, \tau_{m}\right) .
$$

Une fonction $\mu(\tau)$ (ou plus généralement $\mu(\Delta)$ ), sera dite relativement de classe $P_{n}$ (ou $C_{n}$ ), lorsqu'il existe une relation $\varphi(\tau, \sigma)$ de classe $P_{n}$ (resp. $C_{n}$ ) telle que

$$
\varphi(\tau, \sigma)(\tau \epsilon \Psi) \equiv[\sigma=\mu(\tau)] .
$$

Les théorèmes du $\mathrm{N}^{0}$ précédent se laissent relativiser comme suit:

Pour que la fonction $\mu(\tau), \tau \in \Psi$, d̀ valeurs complexes soit de classe $P_{n}(n>0)$ relativement $d \Psi$, il faut et il suffit que chacune des fonctions $\mu^{1}(\tau), \mu^{2}(\tau), \ldots$ le soit.

Fundamenta Mathematicae. T. XxviII. 
Car, on a

$$
\begin{gathered}
{\left[\sigma=\mu^{k}(\tau)\right] \equiv \sum_{\Delta}\left(\Delta^{k}=\sigma\right)(\Delta=\mu(\tau)) \equiv \sum_{\Delta}\left(\Delta^{h}=\sigma\right) \varphi(\tau, \Delta)(\tau \in \Psi F),} \\
{[\Delta=\mu(\tau)] \equiv \prod_{k} \sum_{\sigma}\left(\Delta^{k}=\sigma\right)\left(\sigma=\mu^{k}(\tau)\right) \equiv \prod_{k} \sum_{\sigma}\left(\Delta^{h}=\sigma\right) \varphi_{k}(\tau, \sigma)(\tau \in \Psi),}
\end{gathered}
$$

où $\varphi(\tau, \Delta)$ et $\varphi_{k}(\tau, \sigma)$ sont des fonctions propositionnelles de classe $P_{n}$ telles que

$$
\varphi(\tau, \Delta)(\tau \in \Psi) \equiv[\Delta=\mu(\tau)] \quad \text { et } \quad \varphi_{k}(\tau, \sigma)(\tau \in \Psi) \equiv\left[\sigma=\mu^{k}(\tau)\right]
$$

Les fonctions propositionnelles

$$
\sum_{\Delta}\left(\Delta^{k}=\sigma\right) \varphi(\tau, \Delta) \quad \text { et } \quad \prod_{k} \sum_{\sigma}\left(\Delta^{k}=\sigma\right) \varphi_{k}(\tau, \Delta)
$$

étant de classe $P_{n}$, on parvient aux conclusions demandées.

Theorème. $\mu(\tau)$ etant une fonction de classe $P_{n}(n>0)$ relativement $\dot{a} \Psi$ et $\Phi$ un ensemble de classe $P_{n}$ (ou $\left.O_{n}\right)$, l'ensemble $\mu^{-1}(\Phi)$ est de classe $P_{n}$ (resp. $O_{n}$ ) relativement d $\not$.

La deuxieme partie du th. du $N^{0} 4$ se relativise d'une fagon analogue.

En effet, $\varphi$ satisfaisant à (2), il vient

$$
[\mu(\tau) \epsilon \Phi] \equiv \sum_{\sigma}[\sigma=\mu(\tau)](\sigma \in \Phi) \equiv \sum_{\sigma} \varphi(\tau, \sigma)\left(\tau_{\epsilon} \Psi\right)(\sigma \in \Phi),
$$

d'où $\mu^{-1}(\Phi)=\Psi \cdot E \sum_{\sigma} \varphi(\tau, \sigma)(\sigma \in \Phi)$, ce qui prouve que, lorsque lá fonction $\mu$ est de classe $P_{n}$ relativement à $\Psi$, il en est de même do l'ensemble $\mu^{-1}(\Phi)$. .

Si $\Phi$ est de classe $C_{n}$, on a, en désignant par $\Phi^{\prime}$ le complémentaire de $\Phi, \mu^{-1}(\Phi)=\Phi-\mu^{-1}\left(\Phi^{\prime}\right)$, d'où la conclusion demandée.

Pour prouver la deuxième partie du théorème, il suffit de démontrer qu'en posant $\nu^{l}(\Delta)=\mu_{l}\left(\Delta^{t}\right)$, la fonction $\nu^{l}$ est de classe $P_{n}$ relativement ä $\Psi^{m}$. Or, on a

$$
\left[\sigma=\nu^{i}(\Delta)\right] \equiv\left[\sigma=\mu_{i}\left(\Delta^{l}\right)\right]\left(\Delta_{\epsilon} \Psi^{m}\right) \equiv \varphi_{i}\left(\Delta^{i}, \sigma\right)\left(\Delta_{\epsilon} \Psi^{m}\right),
$$

où $\varphi_{i}(\tau, \sigma)$ est de classe $P_{n}$ et $\varphi_{i}(\tau, \sigma)(\tau \in \Psi) \equiv\left[\sigma=\mu_{i}(\tau)\right]$. Selon le théor. du No $4, \varphi_{i}\left(\Delta^{i}, \sigma\right)$ est encore de classe $P_{n}$ (puisque la fonction $\Delta^{i}$ est analýtique), d'où la conclusion demandée.

Corollaire. La superposition de deux fonctions relativement de classe $P_{n}$ est une fonction relativement de classe $P_{n}$.

Plus précisément: nous supposons que $\nu(\sigma)$ ot $\mu(\tau)$ sont définies sur l'ensemble $\Psi$ et que $\mu(\tau) \dot{\epsilon} \Psi$.
Soient $\varphi$ et $\psi$ les prolongements de $\mu$ et $\nu$ de classe $P_{n}$ :

Il vient

$$
\begin{aligned}
& \varphi(\tau, \sigma)(\tau \in \Psi) \equiv[\sigma=\mu(\tau)] \\
& \psi(\sigma, \varrho)(\sigma \in \Psi) \equiv[\varrho=\nu(\sigma)] .
\end{aligned}
$$

$$
\begin{gathered}
{[\varrho=\nu \mu(\tau)] \equiv \sum_{\sigma}[\varrho=\nu(\sigma)][\sigma=\mu(\tau)] \equiv \sum_{\sigma} \varphi(\tau, \sigma)(\tau \in \Psi) \psi(\sigma, \varrho)(\sigma \in \Psi) \equiv} \\
\equiv\left\{\sum_{\sigma} \varphi(\tau, \sigma) \psi(\sigma, \varrho)\right\}(\tau \in \Psi),
\end{gathered}
$$

le terme $(\sigma \epsilon \Psi)$ pouvant être omis, puisque les conditions $\varphi(\tau, \sigma)$ et $(\tau \in \Psi)$ impliquent que $\sigma=\mu(\tau) \in \Psi$.

6. Notion de limite. Appelons le type ordinal $\tau$ limite d'une suite $\Delta$ de types ordinaux $\Delta^{1}, \Delta^{2}, \ldots$ lorsqu'on a $\Delta^{n} \leqslant \tau$, quel que soit $n$, tandis qu'à chaque $\sigma<\tau$ correspond un $n$ tel que $\sigma<\Delta^{n}$. En symboles: lorsque

$$
\prod_{n}\left(\Delta^{n} \leqslant \tau\right) \cdot \prod_{\sigma<\tau} \sum_{n}\left(\sigma<\Delta^{n}\right)
$$

le symbole $\lim _{n=\infty} \Delta^{n}$ n'étant défini d'ailleurs que pour certaines suites (nommées convergentes).

$\mathrm{Si}$, en particulier, la suite $\Delta$ se compose de nombres ordinaux, elle est convergente et elle converge vers un nombre ordinal; la définition de limite que nous venons de donner est alors conforme à la définition habituelle. La relation entre $x$ et $\Delta$ définie par (1) dans le domaine des types ordinaux arbitraires étant analytique, on en conclut que la fonction $\mu(\Delta)=\lim _{n=\infty} \Delta^{n}$ est relativement analytique par rapport à l'ensemble des suites de nombres ordinaux.

\section{§ 2. Nombres ordinaux.}

7. Notions, ,élémentaires ${ }^{6}$. Passons, à présent, au cas le plus important où les variables $\tau$, $\sigma$ etc. parcourent l'ensemble des nombres ordinaux $<\Omega$. Nous les désignerons d'habitude par $\alpha, \beta, \gamma, \ldots$ et leur ensemble par $\Theta$. Nous dirons, pour abréger, qu'une notion (ensemble, fonction, fonction propositionnelle) est relativement de classe $P_{n}$ (ou $C_{n}$ ) lorsqu'elle l'est par rapport à l'ensemble des nombres ordinaux; autrement dit, l'ensemble $\Psi$ du $N^{0} 4$ - sera supposé identique à $\mathcal{O}$ (respectivement à $\mathcal{\Theta}^{n}$ ou à $\left.\mathcal{O}^{*_{0}}\right)$. 
Définition. Toute notion (ensemble, fonction, fonction propositionnelle) qui est simultanément de olasse (JA ot relativement analytique sera dite élémentaire.

Ainsi, par exemple, l'ensemble de tous les nombres ordinaux $<\Omega$ est élémentaire, puisqu'il est de classe $O A$ (ajoutons qu'il n'osti pas borelien!) ${ }^{1}$ ). Comme on verra, les notions de la théorio des nombres ordinaux, habituellement considérées, sont élémentaires.

Théorème 1. Les ensembles élémentaires (plus généralement, les fonctions propositionnelles elémentaires) constituent un corps borelien; cela veut dire que la différence, ainsi que la somme et le produit dénombrables d'ensembles élémentaires, sont des ensembles ellémentaires.

En effet, $\Phi$ étant relativement analyticque par rapport ì $w$, $\left(=\Theta\right.$ ou $\Theta^{n}$ ou $\Theta^{\$ *}$ ) il existe un ensemble analytique $\Xi$ tol que $\mathscr{D}=\mathbb{Z} \Psi$. Done $\Psi-\Phi=\Psi-Z$, ce qui prouve que $\Psi F-\Phi$ est de classes $O A$, puisque $\Psi$ est de classe $C A$. En même temps, $\Psi$ - $\Phi$ est relativement analytique, puisque le complémentaire de $\Phi$ est analytique (l'ensemble $\Phi$ étant supposé de classe $C A$ ).

La deuxième partie du théorème résulte directement de l'additivité et de la multiplicativité des classes $A$ et $C A$.

On voit ainsi que les ensembles élémentaires peuvent être definis comme sous-ensembles $\Phi$ de $\Psi$ qui, de même que II- $\Phi$, sont de classe $O A$.

Le théorème du $\mathrm{N}^{0} 5$ implique directement le théorème suivant.

Theorème 2. $\mu(\beta)$ etant une fonction relativement analytique dont les arguments et les valeurs appartiennent da $\Theta\left(o u \quad \Theta^{n}\right.$ ou $\left.\Theta^{N_{n}}\right)$ et $\Phi$ étant un ensemble élémentaire, l'ensemble $\mu^{-1}(\Phi)$ est elémentaire. En particulier, si les fonctions $\mu_{1}(\beta), \ldots, \mu_{m}(\beta)$ sont relativement analytiques et si $r\left(\gamma_{1}, \ldots, \gamma_{m}\right)$ est une fonction propositionnelle elémentaire, il en est de même de la fonction propositionnelle $\varphi\left[\mu_{1}\left(\beta_{1}\right), \ldots, \mu_{m}\left(\beta_{m}\right)\right]$.

On n'a qu'à tenir compte du fait que, $\Phi$ étant un ensemble

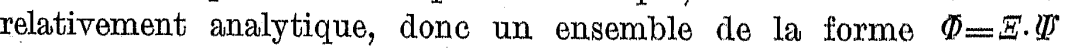
où $\mathbb{Z}$ est analytique, il vient $\mu^{-1}(\Phi)=\mu^{-1}(\mathbb{E})$, puisque les valeurs de la fonction $\mu$ appartiennent à $\Psi$ par hypothèse.

Nous en déduirons plusieurs conséquences importantes en nous appuyant sur un des théorèmes fondamentaux de la théorie des nombres ordinaux, à savoir, sur la trichotomie:

$$
\text { pour } \alpha, \beta<\Omega \text { on a }\{\alpha=\beta\}=\{\alpha \ll \beta \nless \alpha\} \text {. }
$$

1) Cf. le renvoi 3, p. 169.

\section{On a d'abord le}

Corollaire 1. L'égalité $\alpha=\beta$ est une relation élémentaire.

Car la relation $\tau<\sigma$ étant analytique et la relation $\tau, \sigma<\Omega$ étant de classe $C A$, la fonction propositionnelle $(\tau \nless \sigma \nless \tau)(\tau, \sigma<\Omega)$ est de classe $C A$. Mais celle-ci équivaut, comme nous venons de dire, à $(\tau=\sigma)(\tau, \sigma<\Omega)$. En d'autres termes, la relation $\alpha=\beta$ est de classe $C A$. Elle est, en outre, relativement analytique, puisque l'égalité $\tau=\sigma$ (dans le domaine des types ordinaux tout entier) est analytique (voir $\mathrm{N}^{0} 2$ ).

D'une façon plus générale, on a le

Corollaive 2. Toute fonction $\mu(\beta)$ relativement analytique est élémentaire.

En effet, substituons dans le théorème: à $\varphi\left(\gamma_{1}, \gamma_{2}\right)$ la relation $\gamma_{1}=\gamma_{2}$ et posons $\mu_{1}(\beta)=\beta$ et $\mu_{2}(\beta)=\mu(\beta)$. La relation $\gamma_{1}=\gamma_{2}$ étant élémentaire, la fonction propositionnelle $\varphi\left(\gamma_{1}, \mu\left(\gamma_{2}\right)\right)$, qui équivaut à $\left[\gamma_{1}=\boldsymbol{\mu}\left(\gamma_{2}\right)\right]$ l'est également.

Corollaires 3. Les inégalités $\alpha \leqslant \beta$ et $\alpha<\beta$ sont des relations élémentaires.

t. La superposition de deux fonctions élémentaires est une fonction élémentaire.

5. Les fonctions $\beta+\gamma, \beta \cdot \gamma, \beta-1, \lim _{n=\infty} \Delta^{n}$ sont élémentaires.

Le corollaire 3 se déduit de l'équivalence

$$
(\alpha \leqslant \beta) \equiv(\beta \nless \alpha) \text {. }
$$

Les corollaires 4 et 5 résultent directement du corollaire du $\mathrm{N}^{0} 5$, du cor. 2 et des théorèmes du $\mathrm{N}^{0} 2$ et 6

8. Définitions par induction transfinie. La forme habituelle des définitions par induction transfinie est la suivante. Etant donnés un nombre transfinie $\alpha$ et une fonction $\varkappa(\xi)$ on définit une nouvelle fonction $\mu(\xi), \xi<\Omega$, par les conditions:
1) $\boldsymbol{\mu}(0)=0$
2) $\mu(\xi+1)=x \mu(\xi)$
3) $\mu(\xi)=\lim _{\eta<\xi} \mu(\eta)$

si $\xi$ est un nombre limite $\neq 0$.

Cette définition implicite détermine en effet la fonction $\mu$, car on démontre sans difficulté qu'il existe une et une seule fonction $\mu$ qui satisfait aux conditions 1 )-3). 
Pour pouvoir appliquer la méthode d'évaluation de la classe projective de la fonction $\mu$, il faut tout d'abord transformer cette définition implicite en une définition explicite. Nous allons démontrer en effet que, pour qu'on ait $\gamma=\mu(\delta)$, il faut et il suffit qu'il existe une suite $\Gamma=\left[\Gamma^{1}, T^{2}, \ldots\right]$ telle qu'en désignant par $\Delta=\left[\Delta^{1}, \Delta^{2}, \ldots\right]$ une suite composée de tous les nombres $\leqslant \delta$, on ait

$$
\begin{array}{ll}
1^{0} & \Gamma^{n}=\alpha \text { si } \Delta^{n}=0, \quad T^{n}=\gamma \text { si } \Delta^{n}=\delta, \\
2^{0} & I^{n}=\varkappa\left(\Gamma^{m}\right) \text { si } \Delta^{n}=\Delta^{m}+1,
\end{array}
$$

$3^{0}$ si $\Delta^{n}$ est un nombre limite $\neq 0, T^{n}$ est la limito de tous les $\Gamma^{k_{i}}$ tels que $\Delta^{k_{i}}<\Delta^{n}$.

En symboles logiques

$$
\begin{aligned}
{[\gamma=\mu(\delta)] \equiv \sum_{\Delta T}\left\{\prod_{n}\left(\Delta^{n} \leqslant \delta\right) \cdot \prod_{\beta<\delta+1} \sum_{n}\left(\beta=\Delta^{n}\right) .\right.} \\
\prod_{n}\left[\left(\Delta^{n}=0\right) \rightarrow\left(T^{n}=\alpha\right)\right]\left[\left(\Delta^{n}=\delta\right) \rightarrow\left(\Gamma^{n}=\gamma\right)\right] . \\
\prod_{m n}\left[\left(\Delta^{n}=\Delta^{m}+1\right) \rightarrow\left(T^{n}=x\left(T^{m}\right)\right)\right] \\
\prod_{n}\left[\left(0 \neq=\Delta^{n} \in L\right) \rightarrow \prod_{h}\left(\left(\Delta^{h}<\Delta^{n}\right) \rightarrow\left(T^{k} \leqslant I^{n}\right)\right) .\right. \\
\left.\left.\cdot \prod_{\xi<T^{n} l} \sum_{l}\left(\xi<T^{l}\right)\left(\Delta^{l}<\Delta^{n}\right)\right]\right\} .
\end{aligned}
$$

Démonstration. Admettons que $\gamma=\mu(\delta)$. Il s'agit de définir les suites $\Delta$ et $T$ de façon à satisfaire aux conditions $11^{0}-3^{0}$, Or, soit $\Delta$ une suite arbitraire composée de tous les nombres $\leqslant \delta$. Posons $\Gamma^{n}=\mu\left(\Delta^{n}\right)$. Il résulte aussitôt des formules 1$)-3$ ) que les conditions $1^{0}-3^{0}$ sont réalisées.

2. Admettons à présent l'existence des suites $\Delta$ et $T$ assujetties aux conditions $1^{0}-3^{0}$. Il s'agit de démontrer que $\gamma=\mu(\delta)$. Nous allons démontrer que, d'une façon plus générale, $T^{n}=\mu\left(\Delta^{n}\right)$ quel que soit $n$. Procédons par induction transfinie par rapport aux nombres $\Delta^{n}$ rangés selon leur grandeur croissante.

Si $\Delta^{n}=0$, les cond. $1^{0}$ et 1 ) entraînent aussitôt

$$
\Gamma^{n}=\alpha=\mu(0)=\mu\left(\Delta^{n}\right) \text {. }
$$

Admettons que $\mu\left(\Delta^{m}\right)=\Gamma^{m}$ et que $\Delta^{n}=\Delta^{m}+1$. Il vient selon. $2^{0}$ et 2) $\Gamma^{n}=\varkappa\left(\Gamma^{m}\right)=\varkappa \mu\left(\Delta^{m}\right)=\mu\left(\Delta^{m}+1\right)=\mu\left(\Delta^{n}\right)$.
Admettons enfin que $\Delta^{n}$ est un nombre limite $\neq 0$, que $\Delta^{k_{1}}, \Delta^{k_{2}}, \ldots$ est la suite de tous les $\Delta^{k_{i}}<\Delta^{n}$ et que $\Gamma^{k_{i}}=\mu\left(\Delta^{k_{i}}\right)$. Par hypothèse (cond. $\left.3^{0}\right): T^{n}=\lim _{i=\infty} T^{k_{i}}$. Il vient $\Gamma^{n}=\lim _{i=\infty} \mu\left(\Delta^{f_{i}}\right)=\lim _{\eta<\Delta^{n}} \mu(\eta)=\mu\left(\Delta^{n}\right)$.
selon 3).

Ceci établi, nous en déduirons le théorème suivant

Théorème. Etant donnée une fonction élémentaire $x(\xi)$, la fonction $\mu(\xi)$ definie par les conditions 1)-3) est aussi élémentaire.

Soit, en effet, $\psi(\tau, \sigma)$ une fonction propositionnelle analytique telle que $\psi(\tau, \sigma)(\tau<\Omega) \equiv[\sigma=x(\tau)]$ et remplaçons $\gamma=" \prime(\delta)$ par $\varphi(\delta, \gamma)$, $\left(\Delta^{n}=\delta\right)$ par $\left(\Delta^{n} \nless \delta \nless \Delta^{n}\right),\left(\Delta^{n}=\Delta^{m}+1\right)$ par $\left(\Delta^{n} \nless \Delta^{m}+1 \nless \Delta^{n}\right),\left(\Delta^{k}<\Delta^{n}\right)$ par $\left(\Delta^{n} \$ \Delta^{k}\right)$ et $\left(\Gamma^{n}=\varkappa\left(\Gamma^{m}\right)\right)$ par $\psi\left(\Gamma^{m}, \Gamma^{n}\right)$.

Les variables $\beta, \gamma, \delta$ etc. parcourant à présent les ty pes ordinaux (et non les nombres ordinaux), la fonction propositionnelle $\varphi(\delta, \gamma)$ se trouve définie dans le domaine des types ordinaux et on constate facilement qu'elle est analytique.

On a, en outre, $\varphi(\delta, \gamma)(\delta<\Omega) \equiv[\gamma=\mu(\delta)]$. Car si l'on suppose que $\delta<\Omega$ et que $\Delta$ et $\Gamma$ satisfont à la condition qui leur est imposée dans la définition de $\varphi(\delta, \gamma), \Delta$ est une suite de nombres ordinaux et en reprenant la partie 2 de la démonstration précédente, on parvient à l'égalité $\gamma=\mu(\delta)$.

Le théorème précédent se généralise comme suit.

Etant données deux fonctions élémentaires $x(\xi, \eta)$ et $\lambda(\eta)$, la fonction $\mu(\xi, \eta)$ définie par les conditions suivantes est elémentaire:
1') $\mu(0, \eta)=\lambda(\eta)$
$\left.2^{\prime}\right) \quad \mu(\xi+1, \eta)=x[\mu(\xi, \eta), \eta]$
$\left.3^{\prime}\right) \quad \mu(\xi, \eta)=\lim _{\xi<\xi} \mu(\xi, \eta)$ pour $\xi$ limite $\neq 0$.

Pour avoir une définition explicite de la relation $\gamma=\mu(\delta, \eta)$, on n'aura qu'à remplacer dans la définition explicite de la relation $\gamma=\mu(\delta)$, donnée tout-à-l'heure: $\alpha$ par $\lambda(\eta)$ et $\varkappa\left(T^{m}\right)$ par $\varkappa\left(\Gamma^{m}, \eta\right)$. On en conclut, comme auparavant, que la fonction $\mu(\delta, \eta)$ est élémentaire.

Voici quelques applications de l'induction transfinie:

1. $x(\xi, \eta)=x(\xi)=\xi+1$ et $\lambda(\eta)=\eta$. On voit aussitôt que, dans ce cas, $\mu(\xi, \eta)=\eta+\xi$.

2. $x(\xi, \eta)=\xi+\eta$ et $\lambda(\eta)=0$. Ici $\mu(\xi, \eta)=\eta \cdot \xi$.

3. $x(\xi, \eta)=\xi \cdot \eta \quad$ et $\lambda(\eta)=1$. Ici $\mu(\xi, \eta)=\eta^{\xi}$. 
D'après le théorème précédent, la puissance $\alpha=\beta^{\gamma^{\prime}}$ est une opération élémentaire (quant à l'addition et la multiplication cela a été démontrée d'une autre façon).

Le théorème implique directement (cf. $\left.\mathrm{N}^{0} 2,6\right)$ ) le

Corollaire. Si $\varkappa(\xi)$ est une fonction élémentaire, l'ensemble des points critiques de la fonction $\mu(\xi)$, c. $\grave{a}$ d. des $\xi$ tels que $\mu(\xi)=\xi$, est élémentaire.

Ainsi, par exemple, si l'on pose dans 2 et $3: \eta=\omega$, on en conclut que les nombres critiques de la fonction $\omega \cdot \xi$ (dont le premier est $\omega^{\omega}$ ) ainsi que de la fonction $\omega^{\frac{\xi}{5}}$ (c. à d. les nombres „ह-iens“") constituent des ensembles élémentaires.

9. Fonctions caractéristiques. La fonction caractéristique d'un ensemble élémentaire est élémentaire.

Soit, en effet, $\Phi$ un ensemble élémentaire de nombres ordinaux (complexes ou non) et $\mu(\alpha)$ sa fonction caractéristique, c. à d. que $\mu(a)=1$ lorsque $a \in \Phi$ et $\mu(\alpha)=0$ lorsque $\alpha \in \mathcal{O}-\Phi$.

En symboles

$$
[\beta=\mu(\alpha)] \equiv[(\beta=1)(\alpha \in \Phi)+(\beta=0)(\alpha \in \Theta-\Phi)] .
$$

La relation $\beta=\mu(\alpha)$ étant évidemment élémentaire, il s'agit de définir une fonction propositionnelle analytique $\varphi(\tau, \sigma)$ telle que $\varphi(\tau, \sigma)(\tau<\Omega) \equiv[\sigma=\mu(\tau)]$. Or, soit $\Phi^{*}$ un ensemble analytique de types ordinaux tel que $\bar{\Phi}=\Phi^{*} \Theta$. On vérifie facilement que la fonction propositionnelle

$$
\varphi(\tau, \sigma) \equiv\left[(\sigma=1)\left(\tau \in \Phi^{*}\right)+(\sigma=0)(\tau \in \Phi)^{\prime}\right]
$$

satisfait à la condition imposée.

10. Fonction, ,péanienne" Comme on sait, il existe une correspondance biunivoque entre l'ensemble de tous les couples $\alpha, \beta<\Omega$ et l'ensemble de tous les nombres $\gamma<\Omega$. Cette correspondance peut être réalisée à l'aide d'une fonction élémentaire. Posons, en effet ${ }^{1}$ ),

$$
\left\{\begin{aligned}
\mu(\alpha, \beta) & =2^{c} \cdot(2 \beta+1) & & \text { si } a \geqslant \omega \text { ou } \beta \geqslant \omega \\
& =2^{\alpha} \cdot(2 \beta+1)-1 & & \text { si } \alpha, \beta<\omega .
\end{aligned}\right.
$$

1) Voir W. Sierpiński, Teorja Mnogości, Warszawa 1928, t. I, p. 215.
En désignant par $\chi(\alpha, \beta)$ la fonction caractéristique de l'ensemble de tous les couples d'entiers positifs $(\alpha, \beta<\omega)$, il vient

$$
\mu(\alpha, \beta)=\chi^{\prime}(\alpha, \beta) \cdot 2^{\omega}(2 \beta+1)+\chi(\alpha, \beta) \cdot\left[2^{\prime \prime}(2 \beta+1)-1\right] .
$$

La puissance étant une fonction élémentaire, on conclut aussitôt des théorèmes des $\mathrm{N}^{0} 7$ et 9 que la fonction $\mu(\alpha, \beta)$ est élémentaire.

La relation $\gamma=\mu(\alpha, \beta)$ définit deux fonctions élémentaires $\nu(\gamma)$ et $\varrho(\gamma)$ telles que $\gamma=\mu[\nu(\gamma), \varrho(\gamma)]$, c. à d. que la fonction complexe $[\nu(\gamma), \varrho(\gamma)]$ est inverse à la fonction $\mu(\alpha, \beta)$.

On a, en effet,

$$
[\alpha=\nu(\gamma)] \equiv \sum_{\beta \leqslant \gamma}\left[2^{\alpha} \cdot(2 \beta+1)=\gamma \geqslant \omega\right]+\left[2^{\alpha} \cdot(2 \beta+1)-1=\gamma<\omega\right]
$$

et la définition de $\beta=\varrho(\gamma)$ s'obtient de celle-ci en remplaçant $\beta \leqslant \gamma$ par $\alpha \leqslant \gamma$.

Pour démontrer que la fonction $v$ (ainsi que @) est élémentaire, il s'agit de définir une fonction propositionnelle analytique $\psi(\gamma, \alpha)$ telle que $\psi(\gamma, \alpha)(\gamma<\Omega) \equiv[\alpha=\nu(\gamma)]$, les variables $\gamma$ et $\alpha$ parcourant tous les types ordinaux).

Or, soit $\varphi(\boldsymbol{\tau}, \sigma)$ une fonction propositionnelle analytique telle que $\varphi(\tau, \sigma)(\tau<\Omega) \equiv\left(\sigma=2^{\tau}\right)$ et posons (toutes les variables étant des types ordinaux):

$\psi(\gamma, \alpha) \equiv \sum_{\sigma, \beta \leqslant \gamma+1}\{(\alpha \leqslant \gamma) \cdot \varphi(\alpha, \sigma) \cdot[(\sigma \cdot(2 \beta+1)=\gamma \geqslant \omega)+(\sigma \cdot(2 \beta+1)-1=\gamma<\omega)] \cdot$

La fonction propositionnelle $\psi(\gamma, \alpha)$ est analytique.

D'autre part, elle est satisfaite en posant $\alpha=\nu(\gamma)$ pour $\gamma<\Omega$. Car $2^{a} \leqslant \mu(\alpha, \beta)+1, \quad \beta \leqslant \mu(\alpha, \beta)+1$ et $\alpha \leqslant \mu(\alpha, \beta)$.

Enfin, il n'existe, outre $\alpha$, aucun autre $\alpha_{1}$ qui satisfasse à la condition $\psi(\gamma, \alpha)$ pour $\gamma$ fixe $<\Omega$. Car, en supposant que les variables $\alpha_{1}, \beta_{1}$ et $\sigma_{1}$ satisfont aussi aux conditions qui leur sont imposées dans la définition de $\psi$, on conclut de l'inégalité $\alpha_{1} \leqslant \gamma$ que $\alpha_{1}<\Omega$, donc que $\varphi\left(\alpha_{1}, \sigma_{1}\right) \equiv\left(\sigma_{1}=2^{\alpha_{1}}\right)$, d'où $2^{\alpha_{1}} \cdot\left(2 \beta_{1}+1\right)=2^{\alpha} \cdot(2 \beta+1)$, done $\alpha=\alpha_{1}$ (la fonction $\mu$ étant biunivoque).

Ceci établi, on en conclut que les fonetions $\nu(\gamma)$ et $\varrho(\gamma)$ sont élémentaires. 